\title{
Development of a Computational Framework on Fluid-Solid Mixture Flow Simulations for the COMPASS Code*
}

\author{
Shuai ZHANG**, Koji MORITA**, Noriyuki SHIRAKAWA*** \\ and Yuichi YAMAMOTO**** \\ **Department of Applied Quantum Physics and Nuclear Engineering, Kyushu University, \\ 744 Motooka, Nishi-ku, Fukuoka, 819-0395, Japan \\ E-mail: morita@nucl.kyushu-u.ac.jp \\ ***The Institute of Applied Energy, \\ 1-14-2 Nishi-shimbashi Minato-ku, Tokyo, 105-0003, Japan \\ ****Japan Systems Corporation, \\ 1-1 Mimami-machi, Kawasaki-ku, Kawasaki, 210-0015, Japan
}

\begin{abstract}
The COMPASS code is designed based on the moving particle semi-implicit method to simulate various complex mesoscale phenomena relevant to core disruptive accidents of sodium-cooled fast reactors. In this study, a computational framework for fluid-solid mixture flow simulations was developed for the COMPASS code. The passively moving solid model was used to simulate hydrodynamic interactions between fluid and solids. Mechanical interactions between solids were modeled by the distinct element method. A multi-time-step algorithm was introduced to couple these two calculations. The proposed computational framework for fluid-solid mixture flow simulations was verified by the comparison between experimental and numerical studies on the water-dam break with multiple solid rods.
\end{abstract}

Key words: Computational Fluid Dynamics, Nuclear Engineering, Liquid-Solid Two-Phase Flow, Numerical Simulation, Free Surface Flow

\section{Introduction}

COMPASS ${ }^{(1)}$ is a new generation safety analysis code based on the moving particle semi-implicit (MPS) method ${ }^{(2)}$ to simulate various local key phenomena in the core disruptive accidents (CDAs) of sodium-cooled fast reactors (SFRs). MPS is a meso-scale, fully Lagrangian, particle method, and has been verified to be a powerful computational tool with lots of applications, especially thermal-hydraulic phenomena with free surfaces.

Numerical studies on fluid-solid mixture flows are needed for understanding the debris flow occurring in CDAs of SFRs. It would be difficult for conventional Eulerian mesh methods to directly simulate such complicated flows since it is necessary to capture or track lots of fluid-solid inter-phases. One of the advantages of the moving particle methods including MPS is that the inter-phase between different materials is always clear. Therefore, it is straightforward to apply the MPS method into multi-phase simulations. Though some studies on the fluid-solid interaction problems with single solid body have already been carried out in the MPS method ${ }^{(3)}$, there is no further investigation on the multi-solid bodies moving in fluids using the MPS method. Several numerical simulations of fluid and solid mixture flows based on the MPS method have been already carried out. For instance, fluid and a single rigid solid block interaction has been successfully modeled and simulated using the MPS method together with the passively moving solid (PMS) model ${ }^{(3)}$. Sakai et 
al. ${ }^{(4)}$ has developed a multi-scale Lagrangian method by employing the distinct element method (DEM) ${ }^{(5)}$, the MPS method and a coarse grain model. This method can simulate solid-liquid two-phase flows with solid particles, of which scale is smaller than the spatial discretization scale for the liquid phase. However, there is no further investigation on the multi-solid bodies moving in fluids using the MPS method. In the present paper, we aim to provide a framework to directly simulate fluid and solid mixture flows, in which the size of a solid body is larger than that of a typical moving fluid particle, by coupling the MPS method and DEM.

In this study, a computational framework for fluid-solid mixture flow simulations is developed for the COMPASS code based on the feasibility study to investigate the applicability of the MPS method to interactions between fluid and multi-solid bodies. In this framework, the PMS model ${ }^{(3)}$ is applied for hydrodynamic interactions between fluid and solids. In addition, a well-known fully Lagrangian numerical method for granular flow simulations, DEM ${ }^{(5)}$ is coupled with the MPS calculation to represent the interaction between solids and solid-wall. However, a typical DEM calculation requires a much smaller time step compared with the MPS method. To cope with this problem, a multiple time-step scheme is developed for the MPS and DEM coupling calculations, with each method using a different time-step size. In addition, the size of a solid body would be much larger than that of a typical moving fluid particle in engineering applications. The present study undertakes a basic investigation by making two-dimensional simulations of solid bodies with uniform shape. Multiple moving particles are used to represent a solid body in the simulations. For discussion convenience, we refer to the MPS particles that compose a solid body as the solid particles in this paper. The DEM calculation is implemented for each solid body, while the MPS calculation is carried out for all moving particles. In the present study, water dam breakage with multiple solid cylinder layers is investigated to verify the proposed framework for fluid-solid mixture flow simulations.

\section{Improvement of Fluid Models}

\subsection{Governing equations}

Governing equations for incompressible flows are the mass and momentum conservation equations as follows

$$
\begin{aligned}
& \frac{D \rho_{l}}{D t}=0 \\
& \frac{D \vec{u}}{D t}=-\frac{(\nabla p)_{l l}}{\rho_{l}}+\frac{\nabla(\mu \cdot \nabla \vec{u})_{l l}}{\rho_{l}}+\frac{\vec{f}_{s l}}{\rho_{l}}+\frac{\vec{f}_{\text {others }}}{\rho_{l}}
\end{aligned}
$$

where $\vec{u}, p$ and $\rho_{l}$ are the velocity, pressure and density of a fluid particle, respectively, $\mu$ is the viscosity coefficient, $(\nabla p)_{l l}$ and $\nabla(\mu \cdot \nabla \vec{u})_{l l}$ are the pressure and viscosity forces within the fluid phase, respectively, $\vec{f}_{s l}$ is the interaction force between fluid and solid phases, and $\vec{f}_{\text {others }}$ are other volume forces, such as gravity.

The governing equations for solid bodies are

$$
\begin{aligned}
& m \frac{D \vec{u}}{D t}=\vec{F}_{c o l}+\vec{f}_{l s} V+\vec{F}_{\text {others }} \\
& I \frac{D \vec{\omega}}{D t}=T_{c o l}+T_{l s}+T_{\text {others }}
\end{aligned}
$$


where $\vec{u}, \vec{\omega}, m, V$ and $I$ are the translation velocity, rotation velocity, mass, volume and inertia of a solid body, respectively. $\vec{F}_{c o l}$ and $T_{c o l}$ are the force and torque, respectively, caused by collisions between solid bodies and collisions of solid bodies with solid walls, $\vec{f}_{l s}$ and $T_{l s}$ are the volume force and torque, respectively, caused by interactions between fluid and solid phases, and $\vec{F}_{\text {others }}$ and $T_{\text {others }}$ are other forces and torques, respectively, for example those due to gravity.

\subsection{DEM model}

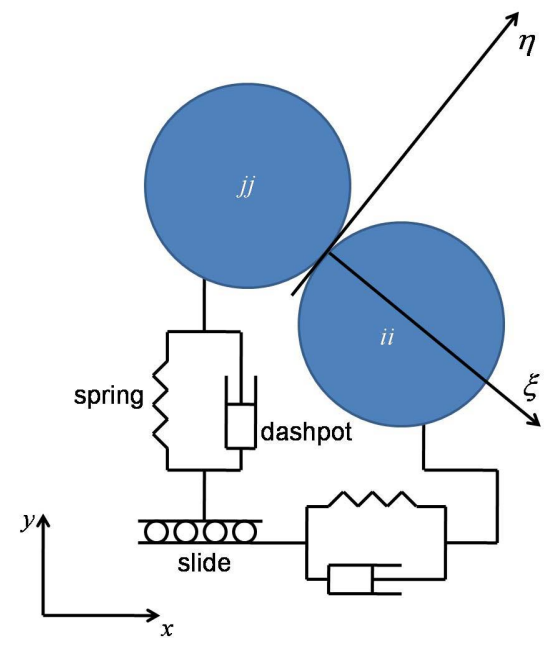

Fig. 1 Schematic description of the DEM model.

In the present study, we formulate the DEM calculation for solid bodies of the same diameter in two-dimensional systems. Therefore, they are modeled as cylinders with a unit length. If the distance between the mass centers of two solid bodies is less than their diameters, they are defined as being in contact. Otherwise, the collision force between two bodies is assumed to be zero. The model of DEM is described schematically in Fig. 1. The collision effects between two solids are modeled by a spring and dashpot, which represent elastic and damping forces, respectively. The slide represents a switch for the maximum static friction force. In addition to the global coordinate $(x, y)$, a local coordinate $(\xi, \eta)$ in normal and tangent directions is constructed for solid $i i$ for convenience of calculation. The two-dimensional collision force of solid body $i i$ received from solid body $j j$, $\vec{F}_{j j \rightarrow i i}=\left(F_{\xi, j j \rightarrow i i}, F_{\eta, j j \rightarrow i i}\right)$, is calculated in DEM as ${ }^{(6)}$

$$
\begin{aligned}
& \left\{\begin{array}{l}
F_{\xi, j j \rightarrow i i}(t)=e_{n}(t)+d_{n}(t) \\
e_{n}(t)=e_{n}\left(t-\Delta t_{D E M}\right)+k_{n} \Delta \xi_{i i, j j} \\
d_{n}(t)=c_{n} \frac{\Delta \xi_{i i, j j}}{\Delta t_{D E M}}
\end{array}\right. \\
& \left\{\begin{array}{l}
F_{\eta, j j \rightarrow i i}(t)=e_{s}(t)+d_{s}(t) \\
e_{s}(t)=e_{s}\left(t-\Delta t_{D E M}\right)+k_{s} \Delta \eta_{i i, j j} \\
d_{s}(t)=c_{s} \frac{\Delta \eta_{i i, j j}}{\Delta t_{D E M}}
\end{array}\right.
\end{aligned}
$$


where $\Delta t_{D E M}$ is the time-step size of the DEM calculation. The following conditions apply to the collision force ${ }^{(6)}$

$$
\begin{array}{llc}
F_{\xi, j j \rightarrow i i}(t)=F_{\eta, j j \rightarrow i i}(t)=0 & \text { for } & e_{n}(t) \leq 0 \\
\left|F_{\eta, j j \rightarrow i i}(t)\right|=\mu \cdot\left|e_{n}(t)\right| & \text { for } & \left|e_{s}(t)\right|>\mu e_{n}(t)
\end{array}
$$

where $F_{\eta, j i \rightarrow i}(t)$ has the same sign as $e_{s}(t)$. Equation (7) states there is no contact effect with negative elastic force, which means that two solids detach from each other. Equation (8) is a switch between static and dynamic friction forces.

In Eqs. (5) and (6), $e_{n}$ and $e_{s}$ are the elastic forces in normal and tangent directions, respectively, $d_{n}$ and $d_{s}$ are the damping forces in normal and tangent directions, respectively, $k_{n}$ and $k_{s}$ are the stiffness in normal and tangent directions, respectively, and $c_{n}$ and $c_{s}$ are the damping coefficients in normal and tangent directions, respectively.

The relationships between the DEM parameters ${ }^{(6)}$ are

$$
\begin{aligned}
& k_{s}=\frac{k_{n}}{2(1+v)} \\
& c_{n}=\alpha_{c n} \cdot 2 \sqrt{m k_{n}} \\
& c_{s}=\frac{c_{n}}{\sqrt{2(1+v)}}
\end{aligned}
$$

where $m$ is the mass of a solid body, $v$ is the Poisson ratio, $\mu$ is the maximum static friction coefficient. The relationship between the global and local coordinates is expressed for $\Delta \xi_{i i, j j}$ and $\Delta \eta_{i i, j j}$, which are the increases in distance between two solid centers in the normal and stress directions, respectively ${ }^{(6)}$

$$
\begin{aligned}
{\left[\begin{array}{c}
\Delta \xi_{i i, j j} \\
\Delta \eta_{i i, j j}
\end{array}\right]=} & M_{i i}\left[\begin{array}{c}
u_{i i}-u_{j j} \\
v_{i i}-v_{j j}
\end{array}\right] \Delta t_{D E M} \\
& +\left[\begin{array}{cc}
0 & 0 \\
\omega_{c, i i} & \omega_{c, j j}
\end{array}\right]\left[\begin{array}{l}
R_{i i} \\
R_{j j}
\end{array}\right] \Delta t_{D E M}
\end{aligned}
$$

where $[u, v]$ and $\omega_{c}$ are the solid's translation velocity vector and rotation velocity, respectively, and $\omega_{c}$ is positive in the anti-clockwise direction. $R_{i i}$ and $R_{j j}$ are the diameters of solids $i i$ and $j j$, respectively, and $M_{i i}$ is the translation matrix between global and local coordinates. In two-dimensional systems, $M_{i i}$ is calculated as

$$
M_{i i}=\left[\begin{array}{cc}
\cos \left(\omega_{c, i i} \Delta t_{D E M}\right) & -\sin \left(\omega_{c, i i} \Delta t_{D E M}\right) \\
\sin \left(\omega_{c, i i} \Delta t_{D E M}\right) & \cos \left(\omega_{c, i i} \Delta t_{D E M}\right)
\end{array}\right]
$$

The collision force acting on solid body $i i$ is calculated as a summation of all forces applied by neighboring solid bodies that are in contact with solid body $i i$

$$
\vec{F}_{c o l, i i}=\sum_{j j} \vec{F}_{j j \rightarrow i i}
$$


It is worth to note that by keeping the no-slip dynamic boundary condition and the pressure Neumann boundary condition on the surface of solid body, the solid-solid interactions and relative movement of solid bodies calculated by DEM is then reflected in the fluid dynamic calculations. Therefore, the hydrodynamic forces acting on solid bodies, i.e. the drag force, the virtual mass force, the Basset force and the lift force, could be directly simulated in the present framework. A detailed coupling algorithm is introduced in the next section.

\subsection{Coupling algorithm}

The MPS method and DEM together with the PMS model are coupled to directly simulate the fluid-solid mixture flows. Since the diameters of solid bodies would be larger than those of the moving particles, multiple moving particles are used to represent one solid body. In addition, the MPS method and DEM applied in the present study use semi-implicit and fully explicit algorithms, respectively. As a result, the characteristic time-step size of the DEM calculation should be much smaller than that of the MPS calculation. To cope with this problem, a multiple time-step algorithm is introduced, which was developed for numerical simulations of fluid-solid mixture flows based on the finite-volume particle method ${ }^{(7)}$. The procedure for coupling the MPS and DEM calculations is described as followings, shown in Fig. 2.

First, all moving particles are assumed as fluid particles and included in the MPS calculation $^{(3)}$. Thereafter, the velocities and pressures of fluid particles are updated. For the moving particles representing solid bodies, the PMS model ${ }^{(3)}$ is applied to calculate the translation and rotation velocities of each solid body

$$
\begin{aligned}
& \vec{u}_{c, i i}^{* *}=\frac{1}{N} \sum_{i=1}^{N} \vec{u}_{i}^{*} \\
& \vec{\omega}_{c, i i}^{* *}=\vec{\omega}_{c, i i}^{n}+\frac{1}{I_{i i}} \sum_{i=1}^{N} m_{i}\left(\vec{r}_{i}^{n}-\vec{r}_{c, i i}^{n}\right) \times\left(\vec{u}_{i}^{*}-\vec{u}_{i}^{n}\right)
\end{aligned}
$$

where $m_{i}$ is the mass of solid particle $i, \vec{u}^{*}$ is the velocity of the solid moving particle after the MPS calculation, $\vec{u}_{c}^{* *}$ and $\vec{\omega}_{c}^{* *}$ are the translation and rotation velocities of solid bodies, respectively, $N$ is the number of the solid particles representing the solid body $i i, \vec{r}_{i}^{n}$ is the position of solid moving particles, which belong to solid body $i i$ in the previous time step, and $\vec{\omega}_{c}^{n}$ is the rotation velocity of solid bodies in the previous time step. The inertia $I$ of the rigid solid body is calculated as

$$
I_{i i}=\sum_{i=1}^{N} m_{i}\left(\vec{r}_{i}^{n}-\vec{r}_{c, i i}^{n}\right)^{2}
$$

and the mass center $\vec{r}_{c}^{n}$ of the solid body in the previous time step is calculated as

$$
\vec{r}_{c, i i}^{n}=\frac{1}{N} \sum_{i=1}^{N} \vec{r}_{i}^{n}
$$

Thereafter, the solid moving particle's position and velocity are modified as

$$
\begin{aligned}
& \vec{r}_{i}^{* *}=\vec{r}_{i}^{n}+M_{i i}\left(\vec{r}_{i}^{n}-\vec{r}_{c, i i}^{n}\right) \\
& \vec{u}_{i}^{* *}=\frac{\vec{r}_{i}^{* *}-\vec{r}_{i}^{n}}{\Delta t_{M P S}}
\end{aligned}
$$


The interaction force between fluid and solid phases at this time step can be thus represented by the pressure and stress forces between solid and fluid particles using the PMS model

$$
\vec{f}_{s l}=-(\nabla p)_{l s}+\nabla(\mu \cdot \nabla \vec{u})_{l s}
$$

If there is only one solid interacting with fluid, the new time-step value for positions and velocities of solid moving particles is then updated using Eqs. (15) and (16). In the present study, the DEM calculation is then performed for each solid body to simulate the collision effects. The increases in distances $\Delta \xi$ and $\Delta \eta$ are calculated from $\vec{u}_{c}^{* *}$ and $\vec{\omega}_{c}^{* *}$ with Eqs. (12), (15) and (16). The collision force between solid bodies is then solved using Eqs. (5), (6) and (14). Correspondingly, the velocities of solid bodies are calculated as

$$
\begin{aligned}
& \vec{u}_{c, i i}^{n, k+1}=\vec{u}_{c, i i}^{n, k}+\Delta t_{D E M} \frac{\vec{F}_{c o l, i i}^{n, k}}{m_{i i}} \\
& \vec{\omega}_{c, i i}^{n, k+1}=\vec{\omega}_{c, i i}^{n, k}+\Delta t_{D E M} \frac{T_{c o l, i i}^{n, k}}{I_{i i}}
\end{aligned}
$$

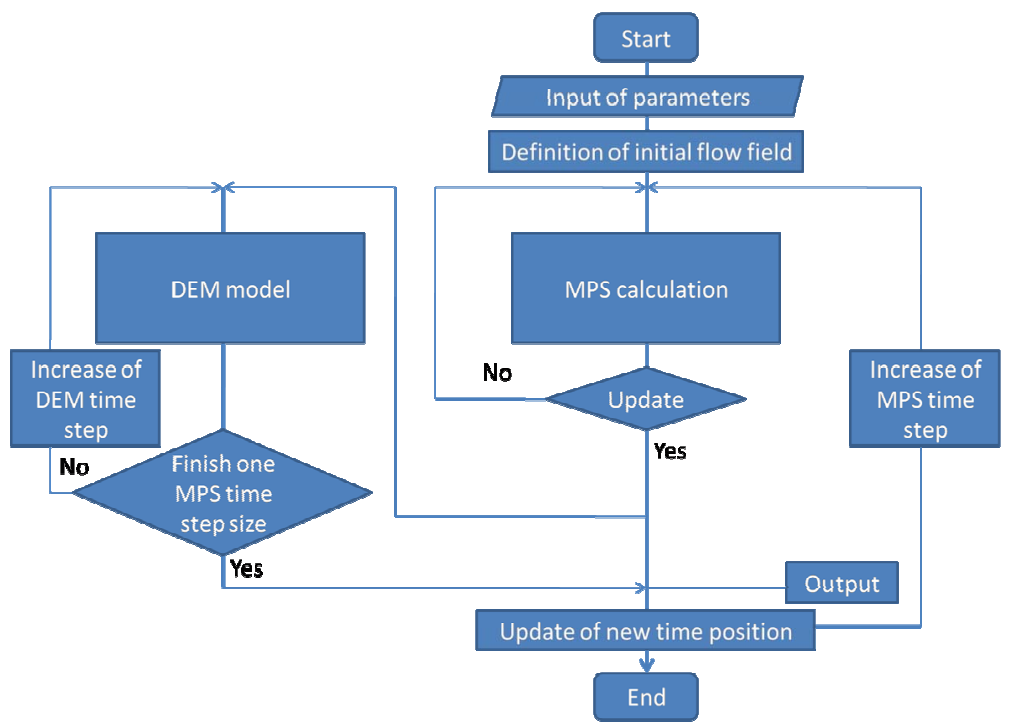

Fig. 2 Flowchart of coupling algorithm for the MPS and DEM calculations.

where $k$ is the number of time steps in the DEM calculation and $n$ is the time step of the MPS calculation, and $m_{i i}$ is the mass of the solid body. The initial values of $\vec{u}_{c}^{n, 0}$ and $\vec{\omega}_{c}^{n, 0}$ are equal to $\vec{u}_{c}^{* *}$ and $\vec{\omega}_{c}^{* *}$, respectively.

Using translation and rotation velocities, the mass center and rotation angle of the solid body are updated as

$$
\begin{aligned}
& \vec{r}_{c, i i}^{n, k+1}=\vec{r}_{c, i i}^{n, k}+\Delta t_{D E M} \vec{u}_{c, i i}^{n, k+1} \\
& \vec{\theta}_{i i}^{n, k+1}=\vec{\theta}_{i i}^{n, k}+\Delta t_{D E M} \vec{\omega}_{c, i i}^{n, k+1}
\end{aligned}
$$

where $\vec{r}_{c}^{n, 0}$ and $\theta^{n, 0}$ are equal to $\vec{r}_{c}^{n}$ and $\vec{\theta}^{n}$, respectively. The position and velocity of the solid particle are updated as

$$
\begin{aligned}
& \vec{r}_{i}^{n, k+1}=\vec{r}_{i}^{n, k}+M_{i i}\left(\vec{r}_{i}^{n, k}-\vec{r}_{c, i i}^{n, k}\right) \\
& \vec{u}_{i}^{n, k+1}=\frac{\vec{r}_{i}^{n, k+1}-\vec{r}_{i}^{n, k}}{\Delta t_{D E M}}
\end{aligned}
$$


where $\vec{r}^{n, 0}$ is equal to $\vec{r}^{n}$.

Finally, after the DEM calculations are performed till $\Delta t_{M P S}=(k+1) \Delta t_{D E M}$ is satisfied, the position and velocity of the solid moving particle are updated as

$\vec{r}_{i}^{n+1}=\vec{r}_{i}^{n, k+1}$

$\vec{u}_{i}^{n, k+1}=\frac{\vec{r}_{i}^{n, k+1}-\vec{r}_{i}^{n, k}}{\Delta t_{D E M}}$

\section{Simulations}

\subsection{Numerical verification of DEM model}

Two calculations were performed to qualitatively verify the developed DEM for the COMPASS code. They were a two-dimensional solid sedimentation through an orifice and collapse of solid cylinder layers. In the former simulation, solid bodies were initially put on a flat plate. As shown in Fig. 3, an orifice was opened in the center of the plate and then solid bodies flow down through the orifice due to the gravity force. The density and diameter of the solid body were set as $2,700 \mathrm{~kg} / \mathrm{m}^{3}$ and $1.0 \mathrm{~cm}$, respectively. The stiffness in the normal and tangent directions, $k_{n}$ and $k_{s}$, were set as $1.0 \times 10^{6} \mathrm{~N} / \mathrm{m}$ and $1.0 \times 10^{5} \mathrm{~N} / \mathrm{m}$, respectively. The damping coefficients in normal and tangent directions, $c_{n}$ and $c_{s}$, were set as $1.0 \times 10^{2} \mathrm{Ns} / \mathrm{m}$ and $10 \mathrm{Ns} / \mathrm{m}$, respectively. The maximum static friction coefficient $\mu$ was chose as 0.3 . The length of the orifice was $6.0 \mathrm{~cm}$. The initial height of the solid cluster $h_{2}$ was $20 \mathrm{~cm}$. The length $L$ and height $H$ of the tank were $40 \mathrm{~cm}$ and $60 \mathrm{~cm}$, respectively. The distance between the orifice and the bottom wall $h_{1}$ was $20 \mathrm{~cm}$. In the simulation, the number of solid bodies was 790. The time step in the DEM calculation was fixed at $1.0 \times 10^{-}$ ${ }^{7}$ s. As shown in Fig. 2, the characteristic transient behavior of the solid movement through the orifice was successfully simulated.

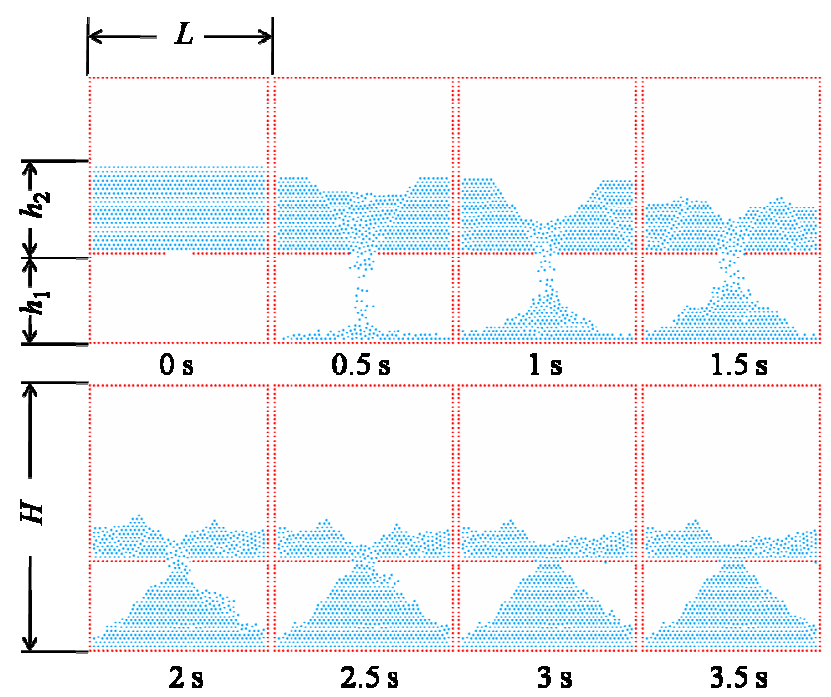

Fig. 3 Snapshot of simulated two-dimensional solid sedimentation through an orifice. 
It is necessary to implement multiple moving particles to represent one solid body since the diameters of solid bodies would be larger than those of the moving particles. A two-dimensional simulation of collapse of solid cylinder layers was performed using DEM to verify the extended DEM. The initial particle arrangement was shown in Fig. 4. Solid cylinders were initially piled up as 20 layers on the left in a tank with a length of $40 \mathrm{~cm}$ and a height of $60 \mathrm{~cm}$. The solid cylinders had a density of $2,700 \mathrm{~kg} / \mathrm{m}^{3}$ and diameter of $1.0 \mathrm{~cm}$. The length and height of the solid cylinder layers were $10 \mathrm{~cm}$ and $20 \mathrm{~cm}$, respectively. In the simulation, one cylinder was represented by 69 moving solid particles. The DEM timestep size was fixed at $1.0 \times 10^{-7} \mathrm{~s}$. In the present DEM calculation, the stiffness and damping coefficients were the same as those used in the previous simulation. Fig. 5 shows the simulated transient behavior of the collapse of solid cylinder layers. The solid cylinders moved downwards due to the gravity and moved to the right wall. After $1.9 \mathrm{~s}$, the solids became still.

Though there were no experimental results available for comparisons, these two simulations using the developed DEM shows characteristic behaviors of the solid bodies. It is necessary for us in the future studies to carry on experiments for comparisons and continue to develop DEM with higher calculation efficiency and the capability for treating with non-uniform solid shapes.

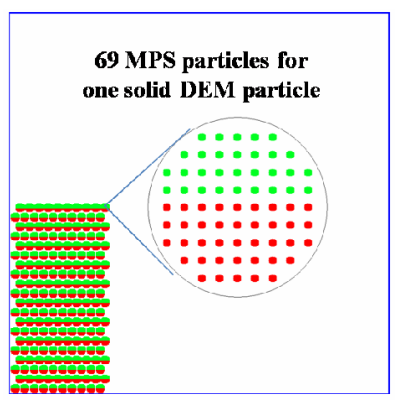

Fig. 4 Initial particle arrangement for simulation of collapse of 20 solid cylinder layers.

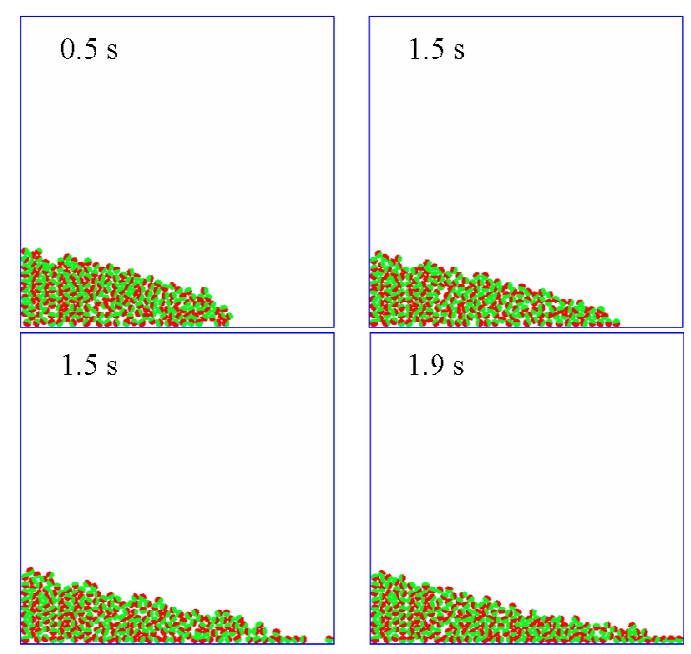

Fig. 5 Transient behavior of collapse of 20 solid cylinder layers.

\subsection{Fluid-solid mixture flow}

The breaking of a water dam involving multiple solid cylinder layers was used to verify the proposed coupling algorithm for the MPS and DEM calculations. For the present 
fluid-solid mixture flow simulations, two-dimensional calculations were performed. As shown in Fig. 6, the length and height of the solid and water mixture column were $6.4 \mathrm{~cm}$ and $12.8 \mathrm{~cm}$, respectively, and five cylinder solid layers were used. The length and height of the tank were both of $26 \mathrm{~cm}$. The density and viscosity coefficient of water were set as $1,000 \mathrm{~kg} / \mathrm{m}^{3}$ and $1.0 \times 10^{-3} \mathrm{~m}^{2} / \mathrm{s}$, respectively. The density and diameter of the solid cylinder were set as $2,700 \mathrm{~kg} / \mathrm{m}^{3}$ and $1.0 \mathrm{~cm}$, respectively.

In the simulation, the initial distance between moving particles was $1 \mathrm{~mm}$. One solid cylinder was represented by 69 moving solid particles. The initial moving particle arrangement is shown in Fig. 6. The MPS and DEM time-step sizes were chosen as $1.0 \times 10^{-}$ ${ }^{4} \mathrm{~s}$ and $1.0 \times 10^{-7} \mathrm{~s}$, respectively. In the present DEM calculation, the stiffness and damping coefficients were the same as those used in the previous simulations.

Figure 7 shows the transient mixture flow behaviors for the simulation in the case of five cylinder layers. The characteristic behaviors of the cylinder movement in the mixture flow were well represented by the simulations using DEM. For convenience, the initial arrangement of the five layers of cylinders in the water dam is separated by a left-upper square, a right-upper oval and a central triangle. A comparison of the mixture flows in the tank at $0.2 \mathrm{~s}$ for the experiment ${ }^{(7)}$ and simulation using DEM is shown in Fig. 8. The experiment was performed using alumina solid cylinders with a length of $9.9 \mathrm{~cm}$ as solid bodies in the water. In both the experiment and simulation, characteristic behaviors are seen in the cylinder movement.

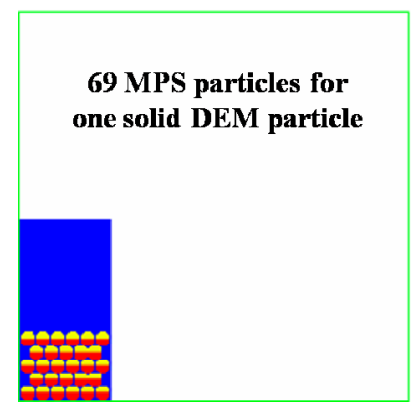

Fig. 6 Initial particle arrangement for simulation of water dam breakage with five solid cylinder layers.

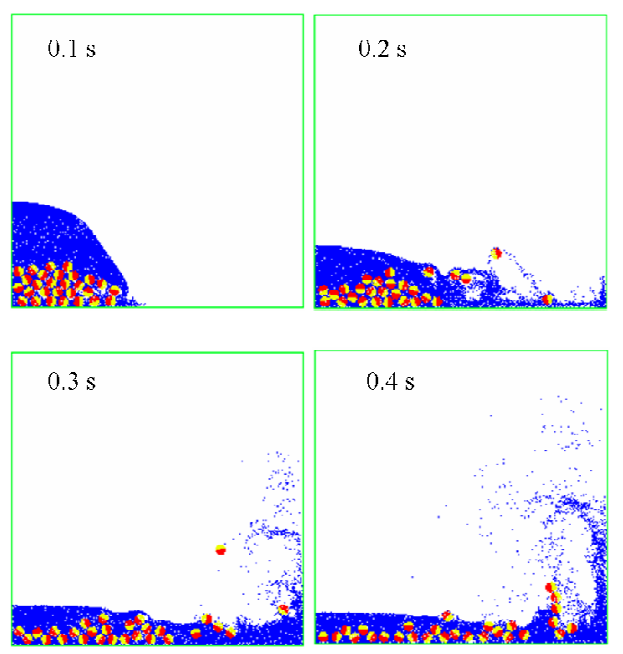

Fig. 7 Transient behavior of water dam breakage with five solid cylinder layers. 
Due to the gravity force, the fluid-solid mixture column would collapse and move down. The following velocities of the solid cylinders were smaller than the water because of the friction between solids and between solid and walls. As a result, the water would impact on the right-upper cylinders. The right-upper cylinders then detached from the cylinder cluster and bounced on the bottom wall. They would arrive at the right wall first. The left-upper cylinders dropped to the left-bottom corner of the tank and then spread laterally. Thereafter, the central cylinders moved together from left to right and maintained their triangular formation till they fully spread on the bottom wall. It is then verified that the COMPASS calculation obtained consistent results with the experiment observations.
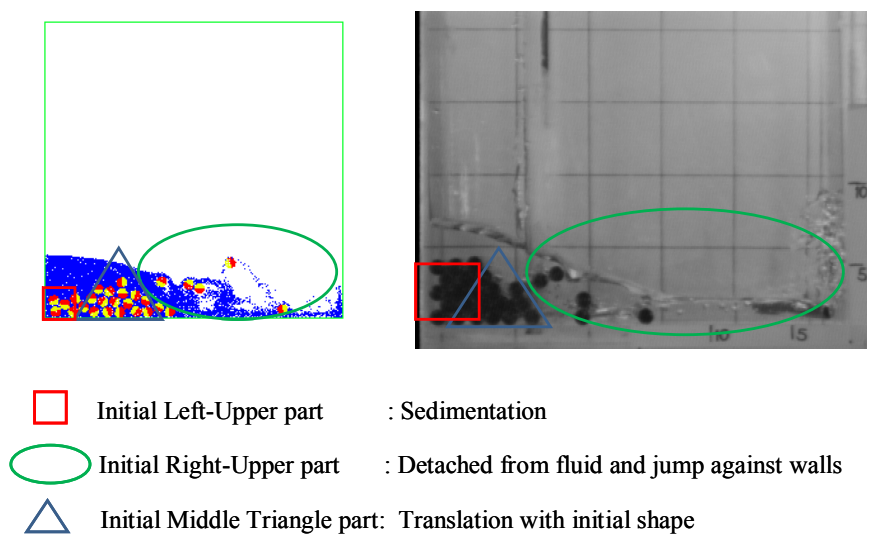

Initial Left-Upper part

: Sedimentation

Initial Right-Upper part

Detached from fluid and jump against walls

Initial Middle Triangle part: Translation with initial shape

Fig. 8 Mixture flow behaviors after the water dam breakage with five cylinder layers at $0.2 \mathrm{~s}$.

\section{Conclusion}

In this study, a computational framework for fluid-solid mixture flow simulations was developed for the COMPASS code. The introduced DEM was used to simulate two typical solid interaction phenomena. The characteristic movements of the solids were successfully simulated. Thereafter, the proposed algorithm was applied to the simulation of a water dam breaking, involving multiple solid cylinder layers. The MPS simulation using DEM reasonably represented the transient behavior of the fluid-solid mixture flow by comparison with the experiments. As a result, it was demonstrated that the present computational framework enhances the capability of the COMPASS code for meso-scale simulations of fluid-solid mixture flow phenomena relevant to CDAs of SFRs. For the future studies, we will optimize the time step size used in DEM calculations by adjusting DEM parameters based on additional experiments and numerical tests. In addition, the DEM calculations for non-uniform solid shapes will be introduced.

\section{Acknowledgements}

The present study was carried out within the task "R\&D of the Next Generation Safety Analysis Methods for Fast Reactors with New Computational Science and Technology" entrusted from the Ministry of Education, Culture, Sports, Science and Technology of Japan. The computation was mainly carried out using the computer facilities at Research Institute for Information Technology, Kyushu University. 


\section{Nomenclature}

$c$ : Damping coefficient

$d$ : Damping force

$e$ : $\quad$ Elastic force

$f$ : $\quad$ External volume force

$F$ : $\quad$ External mass force

I: Inertia

$k$ : $\quad$ Stiffness

$m: \quad$ Mass

$M: \quad$ Translation matrix

$N: \quad$ Number of solid particles

p: $\quad$ Pressure

$r: \quad$ Position coordinate of a particle

$R: \quad$ Radius

$t$ : Time

T: $\quad$ Torque

$u$ : Velocity

$V: \quad$ Volume

\section{Greek Symbols}

$\Delta t: \quad$ Time step size

$\mu: \quad$ Viscosity coefficient/Maximum static friction coefficient

$v: \quad$ Poisson ratio

$\rho$ : $\quad$ Mass density

$\xi$ : Local coordinate in normal direction

$\eta: \quad$ Local coordinate in tangent direction

$\omega: \quad$ Solid rotation velocity

\section{Subscript}
$c$ : $\quad$ Solid mass center
DEM: DEM calculation
$i, j: \quad$ Particle number
$i i, j j: \quad$ Solid body number
$l$ : $\quad$ Liquid phase
MPS: MPS calculation
$n: \quad$ Normal direction
$s$ : Solid phase/Tangent direction

\section{Superscript}

$k$ : $\quad$ Time step in DEM calculation

$n$ : $\quad$ Time step in MPS calculation

\section{References}

(1) Koshizuka, S., Next Generation Safety Analysis Methods for SFRs, (1) Brief Introduction of the Project and Basic Study for Algorithm of Particle Method, 
Proceedings of the 17th International Conference on Nuclear Engineering (ICONE17), (2009-7) ICONE17-75556.

(2) Koshizuka, S. and Oka, Y., Moving-particle Semi-implicit Method for Fragmentation of Incompressible Fluid, Nuclear Science and Engineering, No.123 (1996), pp.421434.

(3) Koshizuka, S., Nobe, A. and Oka, Y., Numerical Analysis of Breaking Waves Using the Moving Particle Semi-implicit Method, International Journal for Numerical Methods in Fluids, Vol.26, Issue 7 (1998), pp.751-769.

(4) Sakai M., Koshizuka, S. and Toyoshima, I., Numerical Simulation of Solid-liquid Flows Involving Free Surface by DEM-MPS Method, Journal of the Society of Powder Technology, Japan, Vol.45 (2008), pp.466-477. (in Japanese)

(5) Cundall, P.A. and Strack, O.D.L., A Discrete Numerical Model for Granular Assemblies, Geotechnique, Vol.29, Issue 1 (1979), pp.47-65.

(6) Gotoh, H. and Sakai, T., Numerical Simulation of Sheetflow as Granular Material, Journal of Waterway, Port, Coastal and Ocean Engineering, Vol.123, Issue 6 (1997), pp.329-326.

(7) Zhang, S., Kuwabara, S., Suzuki, T., Kawano, Y., Morita, K. and Fukuda, K., Simulation of Solid-fluid Mixture Flow Using Moving Particle Methods, Journal of Computational Physics, Vol.228 (2009), pp.2552-2565. 